\title{
A heuristic approach for short-term operations planning in a catering company
}

\author{
Farahani, Poorya; Grunow, Martin; Günther, H.O.
}

Published in:

IEEE International Conference on Industrial Engineering and Engineering Management

Link to article, DOI:

10.1109/IEEM.2009.5372966

Publication date:

2009

Document Version

Publisher's PDF, also known as Version of record

Link back to DTU Orbit

Citation (APA):

Farahani, P., Grunow, M., \& Günther, H. O. (2009). A heuristic approach for short-term operations planning in a catering company. In IEEE International Conference on Industrial Engineering and Engineering Management IEEE. https://doi.org/10.1109/IEEM.2009.5372966

\section{General rights}

Copyright and moral rights for the publications made accessible in the public portal are retained by the authors and/or other copyright owners and it is a condition of accessing publications that users recognise and abide by the legal requirements associated with these rights.

- Users may download and print one copy of any publication from the public portal for the purpose of private study or research.

- You may not further distribute the material or use it for any profit-making activity or commercial gain

- You may freely distribute the URL identifying the publication in the public portal

If you believe that this document breaches copyright please contact us providing details, and we will remove access to the work immediately and investigate your claim. 


\title{
A Heuristic Approach for Short-Term Operations Planning in a Catering Company
}

\author{
P. Farahani ${ }^{1}$, M. Grunow ${ }^{1}$, H. O. Günther ${ }^{2}$ \\ ${ }^{1}$ Department of Management Engineering, Technical University of Denmark, Lyngby, Denmark \\ ${ }^{2}$ Department of Production Management, Technical University of Berlin, Berlin, Germany \\ (pf@man.dtu.dk, grunow@man.dtu.dk, hans-otto.guenther@tu-berlin.de)
}

\begin{abstract}
Certain types of food such as catering foods decay very rapidly. This paper investigates how the quality of such foods can be improved by shortening the time interval between production and delivery. To this end, we develop an approach which integrates short-term production and distribution planning in a novel iterative scheme. The production scheduling problem is solved through an MILP modeling approach which is based on a block planning formulation complemented by a heuristic simplification procedure. Our investigation was motivated by a catering company located in Denmark.

The production configuration and the processes assumed in our numerical experiments reflect real settings from this company. First numerical results are reported which demonstrate the applicability of the proposed approach.
\end{abstract}

Keywords - Production scheduling, block planning, vehicle routing, catering industry, food quality

\section{INTRODUCTION}

Caterers are concerned with the provision of prepared food and drinks ready for consumption away from home [1]. The quality of the products is not only determined by the production process. It is also strongly influenced by the coordination between the production and the distribution environments. To ease this coordination, the food preparation sites are usually directly connected to the customers with a fleet of vehicles operated by the caterer.

However, the analysis of the practices at a Copenhagenbased caterer shows that today the production and distribution stages are planned independently, causing large operational and quality problems. Therefore, in this paper, we are investigating how an integrated planning approach can be used to harmonize the decisions made on production and distribution aiming at an improved quality of the delivered products at the customer location. Reference [2] is the only other paper which explicitly investigates such coordination, however, with a completely different focus on mid-term production planning decisions.

The remainder of this paper is organized as follows: In the next section, an iterative framework for the integrated problem is proposed. Section 3 describes the production environment in greater detail and presents a simplification strategy for production scheduling which is based on a block planning formulation and a heuristic batching method. In section 4, mixed integer linear programming (MILP) models are developed for the production and transportation problems at hand. The results of a numerical study are presented in section 5 and, finally, conclusions are drawn and further research directions are discussed in the last section.

\section{ITERATIVE SOLUTION FRAMEWORK}

The quality of the produced products continuously degrades with time, until the order is delivered. Therefore, there is a tight connection between transportation and production scheduling decisions on the quality of the delivered order. However, due to the complexity of the production scheduling and transportation problems, modeling the entire problem as a single mathematical model does not lead to a tractable formulation. Therefore, we designed a novel iterative framework to handle these problems.

Production decisions include the completion time for individual orders. Transportation decisions involve vehicle routes determining when each order is picked up from the production site (departure time) and its delivery time. Clearly, an order cannot be picked up by a vehicle unless its production is completed.

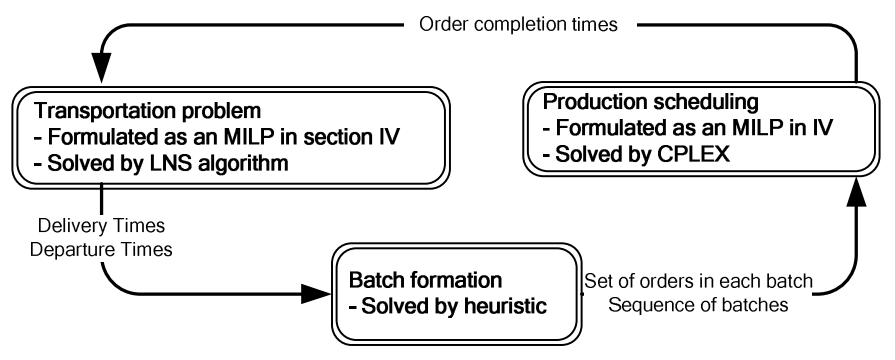

Fig. 1. Solution methodology

Fig. 1 describes the iterative solution framework used to solve the integrated problem. In principle, it is possible to start production at a very early time to have all products ready by the time the vehicles are scheduled to depart from the production site. Hence, we can first solve the distribution problem, knowing that our initial solution will not lead to infeasibility for the production scheduling problem.

In this first iteration, every order's delivery time and the departure time of the vehicle carrying that order are determined. This information is used to generate constraints for the production scheduling problem. Also successive iterations during which delivery and departure times are updated, will not lead to an infeasible production scheduling problem. But any update of the delivery and departure times creates a potential for improving the quality of the delivered orders by rescheduling them. Similarly, in a solution to the production scheduling problem the completion time of the orders provides a potential for rerouting the vehicles to 
improve the quality of the delivered orders without increasing total transportation costs.

As a stopping criterion for iterating between distribution and production scheduling, we compare the objective function value of the respective problem with the value obtained in the previous iteration. If there is no improvement, the procedure is terminated.

The two main constituents of this approach are the solution algorithm for the production scheduling problem and the solution algorithm for the distribution problem (cf. Fig. 1 ). The latter is a typical vehicle routing problem with time windows (VRPTW). In the case which provides the background of this investigation, a fixed number of homogenous vehicles is used. In contrast to standard productiondistribution problems, the quality improvement of the delivered foods is included into the objective function (cf. the model formulation in section IV of this paper). For the solution to this problem, we use a large neighborhood search (LNS) algorithm, which has proven to work very efficiently in tightly constrained problems such as the one studied here [3]. To solve the production scheduling problem, we first apply a heuristic batching method which significantly simplifies the problem (cf. section III) and then model the resulting simplified problem in an MILP formulation based on the concept of block planning which (cf. section IV) is then solved by use of standard software (CPLEX).

\section{CATERING PRODUCTION SCHEDULING}

In this section, the production environment and the heuristic batching method, which works as a preprocessing procedure to the solution of the MILP model formulation, are explained. The general configuration of the production environment is as follows:

- All products are produced at a single production site with several identical ovens. These ovens form the bottleneck of the production system.

- A set of known customer orders arrives daily and has to be processed and delivered within time-windows specified by the customers.

- Every order encompasses only one type of food menu.

- The quality decay of food orders starts right after the completion time.

- Food quality is calculated at the time of delivery.

- Orders can be combined to be produced together in a batch. However, only those orders are allowed to be put in the oven together, which have a similar temperature requirement and a similar processing time. In combining orders, the oven capacity must be respected.

- There can be idle time between two successive batches in an oven. This is accepted to avoid premature production which may result in unacceptable quality decay.

The aim in production scheduling is twofold: (1) minimization of the quality decay, expressed as the time interval between order completion and delivery times, and (2) minimization of total setup costs. The setup costs result from heating up (and cooling down) of the ovens. Different orders need to be processed at different temperatures. Total setup costs hence depend on the sequence of orders at each oven. Such scheduling problems are known to be NP-hard [4]. However, this problem can be significantly simplified by incorporating specific conditions frequently observed in real world cases.

In consumer goods industries manufacturers often consider a fixed sequence of production setups based on human expertise or technological conditions. For instance, in food processing industries, production managers often sequence from the less intensive taste of a food product to the stronger or from the brighter color of a product to the darker. Such production policy is known in practice as block planning (sometimes called production wheel policy) [5]. Applications of the block planning concept have not been widely investigated in literature, although this policy is easy to implement and provides decision makers with managerial insights [6]. One important aspect in this approach is to define a pre-set sequence of setups based on human expertise, and then to construct a hierarchical structure for planning the production operations based on such a fixed setup sequence [7].

In this paper, we use a block planning approach to formulate the production scheduling problem since it not only reflects the practical settings of our application but also significantly reduces the complexity of the problem. Since heating up the ovens is much quicker than cooling them down, orders are scheduled on the ovens based on their temperature level requirements in an ascending order. In our case, a block is hence defined as the sequence of all required temperature levels in ascending order. These blocks are applied to all lines.

Considering such a temperature pattern, total setup costs of an oven can be seen as the costs for heating up the oven to its highest temperature level realized for processing the assigned orders. Obviously, such an objective will result in the most economical schedules. If a temperature level is used for an order $A$ on a line, then the processing of another order $B$ at a lower temperature level on the same line will not have any impact on the setup costs. However, order $B$ must be produced before order $A$ and this might lead to preparing it far before its delivery time. Therefore, the quality decay is also included in the objective function which keeps finishing times of orders close to their delivery times.

The second step of block planning is to construct a hierarchical plan based on the defined blocks. The main idea in developing the hierarchical structure is to decide on which block components to be realized on each line, and to form the production schedule based on such settings.

In this regard, we developed a two stage hierarchical formulation. In one stage, assignment decisions are made on which block components (temperature levels) to be realized on which lines. The other stage is about combining individual orders into production batches and scheduling the generated batches at the realized temperatures.

What we observed was that even after defining blocks, the problem is still too complex to be solved in our iterative framework at a reasonable time. Therefore, the following heuristic batching procedure was designed based on the 
definition of temperature patterns which significantly simplifies the problem without impairing the quality of the final solution.

The procedure described in Table I determines the orders to be included in a batch. The resulting batches cannot be scheduled on the same oven unless their sequence is respected. This significantly reduces the solution space. The production scheduling problem is thus reduced to scheduling a considerably smaller number of entities (batches vs. orders).

TABLE I.

HEURISTIC BATCHING ALGORITHM

\begin{tabular}{cc}
\hline HEURISTIC BATCHING ALGORITHM \\
\hline 1 & Function Batching ( $i$ in nodes) \\
2 & List: $L=$ all orders are sorted as follows: \\
3 & Sort based on their temperatures in ascending order \\
4 & For orders with similar temp. requirements: \\
5 & Sort based on their delivery times in ascending order \\
6 & While $|L|>0$ do \\
7 & Array: batch $\rightarrow$ batch.Load $=0$ \\
8 & $i=$ First order in $L$ \\
9 & Assign $i$ to batch and remove $i$ from $L$ \\
10 & Set $i$. DepartureTime as batch.DepartureTime \\
11 & Add $i$.Demand to current batch.Load \\
12 & While $|L|>0$ do \\
13 & $j=$ First order in $L$ with the same temp. and the \\
& same processing time as $i$ \\
14 & if $(j$. DeliveryTime $<i$.Delivery Time + threshold) $\&$ \\
& (j. DepartureTime $<i$. DepartureTime + threshold) $\&$ \\
& (batch.Load $+j$. Demand $<$ Capacity) \\
15 & Assign $j$ to batch \\
16 & Remove $j$ from $L$ \\
17 & else $\quad$ break \\
18 & end while \\
19 & end while \\
20 &
\end{tabular}

Fig. 2 illustrates how our approach works for a simple example of 11 orders, 3 temperature levels and 3 ovens. Transportation between every two locations is assumed to take 3 units of time and the threshold parameter for batching is considered to be 5. Distribution decisions including vehicle routes and visiting time of each customer are obtained by solving the transportation problem formulated in the next section using LNS. Afterwards, orders are combined into batches through the heuristic algorithm of Table I and then the formed batches are scheduled on different lines by solving the model presented in the next section.

\section{MODEL FORMULATIONS}

In this section, first, a mixed-integer linear formulation and its explanation are presented for the production scheduling problem which is solved once the batching heuristic has been applied. Then, a mixed integer linear formulation for the transportation problem is provided.

The notation used in formulating the production scheduling problem is as follows:

Indices and sets:

- $l \in L \quad$ Production lines (ovens)

- $h \in H$ Temperature levels: $\{1, \ldots, n h\}$ (nh number of temperature levels)

- $k \in J \quad$ Set of batches

- $k \in J_{h}$ Batches requiring temperature level $\mathrm{h}$

- $\quad i, j \in J_{k}^{\prime}$ Orders of batch $k$

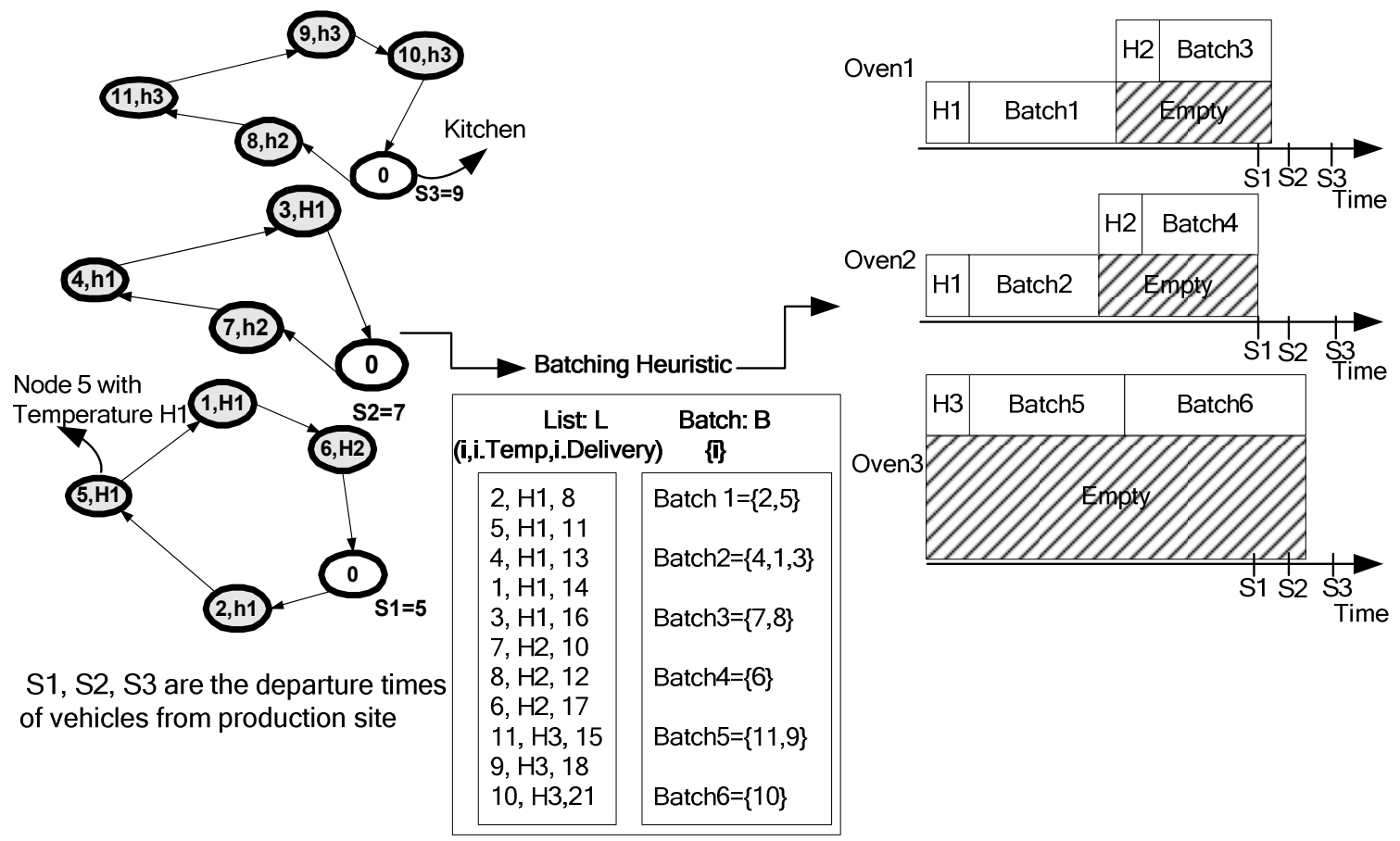

- $\quad$ Fig. 2. Batching heuristic 
Parameters:

- $\alpha_{h_{k}}$ Time required for increasing the oven temperature from level $h_{k}-1$ to $h_{k}$

- $B_{i} \quad$ Delivery time of order $i$

- $D_{k}$ Due date for production of batch $k$ (the departure time of vehicle that carries orders of batch $k$ )

- $\theta_{i} \quad$ Quality decay rate of order $i$ per unit of time

- $h_{k}$ Temperature level of batch $k$

- $\quad c_{h, l}^{\prime}$ Setup cost for realizing temperature $h$ on line $l$

- $\quad M \quad$ Sufficiently large number

- $W_{i}$ Relative weight of objective function $i$

Decision variables:

- $Y_{h, l}=1$ if temperature level $h$ is realized on line $l$

- $Z_{k, l}=1$ if batch $k$ is realized on line $l$

- $F_{k, l} \quad$ Completion time of batch $k$ on line $l$

- $F_{k} \quad$ Completion time of batch $k$

- $S C_{l} \quad$ Setup costs on line $l$

Model formulation:

$$
\operatorname{Min} W_{1} \cdot \sum_{l \in L} S C_{l}+W_{2} \cdot \sum_{k \in J} \sum_{i \in J_{k}^{\prime}}\left(B_{i}-F_{k}\right) \cdot \theta_{i}
$$

S.T.

$$
\begin{array}{ll}
\sum_{k \in J_{h}}^{S . T} Z_{k, l} \leq M \cdot Y_{h, l} & \forall h \in H, \forall l \in L \\
\sum_{l \in L}^{Z_{k, l}=1} & \forall k \in J
\end{array}
$$$$
F_{k, l} \geq F_{k-1, l}+T_{k} \cdot Z_{k, l} \quad \forall k \in J, \forall l \in L \mid h_{k}=h_{k-1}
$$$$
F_{k, l} \geq F_{k-1, l}+\alpha_{h_{k}}+T_{k} \cdot Z_{k, l}
$$$$
\forall k \in J, \forall l \in L \mid h_{k} \neq h_{k-1}
$$$$
F_{k} \leq F_{k, l}+M \cdot\left(1-Z_{k, l}\right) \quad \forall k \in J, \forall l \in L
$$$$
F_{k} \leq D_{k} \quad \forall k \in J_{h}, \forall h \in H, \forall l \in L
$$$$
S C_{l} \geq c_{h, l}^{\prime} \cdot\left(Y_{h, l}-\sum_{m=h+1}^{n h} Y_{m, l}\right)
$$$$
\forall h \in H, \forall l \in L
$$$$
Y_{h, l} \in\{0,1\}, Z_{k, l} \in\{0,1\} \quad \forall h \in H, \forall k \in J, \forall l \in L
$$$$
F_{k, l} \geq 0, F_{k} \geq 0, S C_{l} \geq 0 \quad \forall k \in J, \forall l \in L
$$

The objective function minimizes the weighted sum of total setup costs and total food decay. Setup costs correspond to the highest temperature level realized at each line (8). Food decay is measured as the time difference between the delivery time of an order and its completion time. Since each order is assigned to a batch, its finishing time is the same as the finishing time of the batch in which it is included. Constraints (2) prevent realization of a batch on a line unless its corresponding temperature level is used on that line. Constraints (3) guarantee that every batch will be realized on one of the lines. Constraints (4)-(5) indicate the relationship between completion time of a batch and the completion time of the preceding batch. Constraints (6) set the completion time of each batch with regard to the line on which the batch is set up. Constraints (7) set an upper bound on the completion time of each batch as the vehicle depar- ture time of the earliest of the included orders. Constraints (9)-(10) define variable domains.

The following notation is used in formulating the transportation problem:

Indices and sets:

- $\quad i, j \in S \cup O \cup \bar{O}$ Orders, Production site (starting and ending depots)

- $v \in V$ Vehicles

Parameters:

- $\quad F_{i}^{\prime}$ Completion time of order $i$ given by the production planning phase

- $\quad c_{i, j}$ Transportation time needed to deliver order to location $j$ after $i$

- Cap Capacity of vehicles

- $q_{i} \quad$ Volume of order $i$

- $d_{i}$ Duration of the delivery process for order $i$

- $e_{i} l_{i}$ Earliest and latest time for delivery of order $i$

- $\quad \bar{M} \quad$ Sufficiently large number

- $W_{i}$ Relative weight of objective function $i$

Decision variables:

- $X_{i, j, v}$ If order $j$ is delivered immediately after order $i$ by vehicle $v$

- $B_{i, v} \quad$ Delivery time of order $i$ by vehicle $v$

The transportation problem is a VRPTW which can be modeled as follows (considering production decisions to be fixed):

Min

$W_{2} \cdot \sum_{i \in S}\left(B_{i}-F_{i}^{\prime}\right) \cdot \theta_{i}+W_{3} \cdot \sum_{v \in V} \sum_{i \in S \cup O \cup \bar{o}} c_{i, j} \cdot X_{i, j, v}$

$\sum_{v \in V} \sum_{i \in S \cup O} X_{i, j, v}=1$

$\forall j \in S$

$\sum_{j \in S} X_{i, j, v}=1$

$\forall i \in O, \forall v \in V$

$\begin{array}{ll}\sum_{i \in S} X_{i, j, v}=1 & \forall j \in \bar{O}, \forall v \in V \\ \sum_{j \in S \cup \bar{O}} X_{i, j, v}-\sum_{j \in S \cup O} X_{j, i, v}=0 & \forall i \in S, \forall v \in V\end{array}$

The objective function minimizes the weighted sum of total transportation costs and total food decay. Constraints (12), (13) and (14) are set covering constraints for orders and production-site nodes. Constraints (15) model the flow balance for every node.

Capacity constraints:

$\sum_{i \in S} q_{i} \sum_{j \in S} X_{i, j, v} \leq \operatorname{Cap}_{v} \quad \forall v \in V$ 
Starting time constraints:

$$
\begin{aligned}
B_{j, v} \geq\left(B_{i, v}+d_{i}+c_{i, j}\right)-\bar{M} \cdot\left(1-X_{i, j, v}\right) & \\
l_{i} \geq B_{i, v} \geq e_{i} & \forall i \in S, \forall v \in V \\
& \forall i \in S \cup O, \forall v \in V
\end{aligned}
$$

Variable domains:

$X_{i, j, v} \in\{0,1\}$

$$
\begin{aligned}
& \forall i \in S \cup O \cup \bar{O}, \forall v \in V \\
& \forall i \in S \cup O, \forall v \in V
\end{aligned}
$$

The VRPTW is known to be NP-hard. In this paper, we use a local search algorithm known as large neighborhood search (LNS) to obtain a solution. A detailed discussion of the use of LNS in routing problems is available in [8].

\section{NUMERICAL RESULTS}

The iterative procedure, heuristic batching and LNS are coded in $\mathrm{VC}++6$. The production scheduling problem is solved using OPL ILOG 6.1.1. All programs are run on a standard PC with Intel P4 3.2GH CPU and 1 GB of RAM. Due to confidentiality of the company's data, we can not reveal any numerical results from the real case. Therefore, we used the Solomon R201 instance [8] and modified the demand data to create specific test problems. Table II shows how each component is gradually changed from the first to the last iteration of the algorithm for 4 different instances we drew from the R201 instance. Our focus on product quality which is the underlying rationale for our integration approach yields a significant reduction in product quality decay of $21 \%$ in the real case compared to the company's planning method where the focus is almost only on costs.

TABLE II

Integration effect on different problem sizes

\begin{tabular}{lllll}
\hline Problem size & 50 & 100 & 150 & 200 \\
Quality decay & $-17 \%$ & $-23 \%$ & $-62 \%$ & $-87 \%$ \\
$\begin{array}{l}\text { Transportation and } \\
\text { production }\end{array}$ & $+2 \%$ & $+3.4 \%$ & $+5.1 \%$ & $+7.9 \%$ \\
\hline
\end{tabular}

In most instances, the stopping criterion is met and the solution is found in 10 or less iterations and within a reasonable computational time of less than $6 \mathrm{~min}$. Another numerical study is done on the effect of increasing product decay rates.

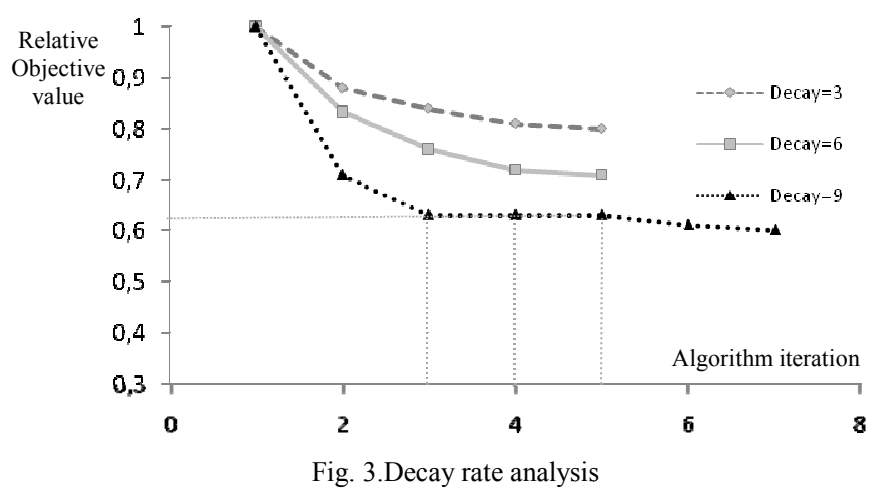

Figure 3 shows that the algorithm works more efficiently when decay rates are increased from 3 to 9 . It is worth noting that such large quality improvement comes at the cost of an only marginal increase in setups and transportation costs.

\section{CONCLUSIONS}

In short-term catering operations, production scheduling and transportation planning need to be harmonized to obtain good product quality. However, both problems are already NP-hard which makes their integration difficult. This paper provides an iterative solution framework for an integration of both problems. Further, a modeling approach to simplify the production scheduling problem based on the block planning concept is developed. The result of the quality focus and the integration of the decisions show significant improvement in the quality of delivered foods in catering business.

One extension to this study is to find an appropriate lower bound for production scheduling against which the influence of applying the block planning approach and the batching heuristic can be measured.

\section{REFERENCES}

[1] Eastham J. F., Ball S. D., Sharples L., Food supply chain management, Oxford: Butterworth-Heinemann, 2001, Chapter 1.

[2] Chen H.K., Hsueh C.F., Chang M.S., "Production scheduling and vehicle routing with time windows for perishable food products," Comp. \& Oper. Res., vol. 36, 2009, pp. 2311 2319.

[3] Ropke S., Pisinger D., “An Adaptive Large Neighborhood Search Heuristic for the Pickup and Delivery Problem with Time Windows," Transp. Sci., vol. 40, no.4, 2006, pp. 455472.

[4] Sun X., Noble J.S., Klein C.M., "Single-machine scheduling with sequence dependent setup to minimize total weighted squared tardiness," IIE Trans., vol. 31, 1999, 113-124.

[5] Lütke Entrup M., Günther H.-O., Van Beek P., Grunow M., Seiler T., "Mixed-integer linear programming approaches to shelf-life-integrated planning and scheduling in yoghurt production," Int. J. of Production Res., vol.43, no. 23, 2005, pp. 5071-5100

[6] Günther H.-O., Grunow M., Neuhaus U., "Realizing block planning concept in make-and-pack production using MILP modelling and SAP APO," Int. J. of Production Res., vol. 44, no. 18-19, 2006, pp. 3711-3726.

[7] Bilgen B., Günther H.-O., "Integrated production and distribution planning in the fast moving consumer goods industry: A block planning application," OR Spectrum, in press

[8] Shaw, P., "A new local search algorithm providing high quality solutions to vehicle routing problems," Technical report, University of Strathclyde, Scotland, 1997

[9] Solomon M.M., "Algorithms for the vehicle routing and scheduling problems with time windows constraints," Oper. Res., vol. 35, 1987, pp. 254-65. 